\title{
Téoros
}

Revue de recherche en tourisme

\section{Les grandeurs et les misères du financement en tourisme}

\section{Léonard Dumas}

Volume 5, numéro 3, novembre 1986

La gestion touristique : les nouvelles technologies

URI : https://id.erudit.org/iderudit/1080541ar

DOI : https://doi.org/10.7202/1080541ar

Aller au sommaire du numéro

Éditeur(s)

Université du Québec à Montréal

ISSN

0712-8657 (imprimé)

1923-2705 (numérique)

Découvrir la revue

Citer cet article

Dumas, L. (1986). Les grandeurs et les misères du financement en tourisme.

Téoros, 5(3), 36-38. https://doi.org/10.7202/1080541ar d'utilisation que vous pouvez consulter en ligne.

https://apropos.erudit.org/fr/usagers/politique-dutilisation/ 


\title{
Les grandeurs et les misères du financement en tourisme
}

\author{
par Léonard Dumas*
}

Plusieurs promoteurs ou proprictaires d'entreprises touristiques ont été, un jour ou l'autre, confrontés à la nécessité de financer leurs projets. Pour nombre d'entre eux, ce fut une découverte: financer un projet n'est pas aussi simple que le laissent entendre les messages publicitaires. En realité, n'obtient pas le financement qui veut et auprès de qui le désire...

L'objet dé cet article est de présenter certaines règles à prendre en considération lors du financement de projets touristiques, notamment en ce qui a trait aux infrastructures hôtelières. En tenant compte des caractéristiques particulières de cette industrie et des modes de financement conventionnels, nous verrons brièvement quelles sont les tendances de t'heure et quels sont les choix qui s'offrent aux éventuels promoteurs.

\section{Caractéristiques de l'industrie hôtelière}

Malgré le fait que les hotels soient classés dans le secteur des services, il n'en demeure pas moins que plusieurs de leurs activitếs s"apparentent aux secteurs manufacturiers. Cette particularité a un impact direct sur les opérations de ce secteur et est à la source de préjugés. On peut "qualifier"' le service d'un hôtel à partir des 6 critères suivants:

Le service est intangible. La conception originale voulant qu"un hơtel soit un endroit où on loue des chambres et serve des repas est beaucoup trop restreinte. En fait, le client d'un hôtel vit une "expérience hôtelière" qui débute au moment même de la réservation pour ne se terminer bien souvent qu'au moment de l'acquittement de la facture, quelques fois plusieurs jours après avoir quitté l'établissement. Le client se souviendra longtemps d'un climat agrétable et d'un accueil personnalise. Dans bien des cas, le critère principal incitant à choisir un établissement plutôt qu'un autre sera justement lå qualité du service qu'il y aura reçu préalablement.

"Lonard Dumas, conseiller sénior, Laventhol \& Hor. wath, firme specialista en hobtellerie, restauration et tourisme.
Le service est périssable. Une chambre non occupée est une chambre perdue. On ne peut en aucun cas faire une réserve de chambres pour la haute saison. Par ailleurs, les aliments sont bien souvent préparés pour une consommation immédiate. Mal= gré ces contraintes, l'hôtelier doit faire face aux fluctuations saisonnières, voire journalières sinon la performance de l'entreprise pourrait en souffrir.

Le service se fonde sur les relations interpersonnelles. Afin de donner le service attendu par la clientèle, il faut non seulement établir de bonnes relations avec les clients, mais également consentir à s'efforcer d'avoir à l'intérieur même de l'entreprise une communication efficace. Ce n'est que par la communication et la coordinattion des efforts des employes que l'entreprise pourra atteindre ses objectifs et satisfaire par le fait mêne les attentes de la clientèle.

La production du service est spontanée. Dans plusieurs entreprises manufacturières, il est possible d'exercer un contróle de la qualité à partir de la chaîne de montagne, évitant ainsi que le client entre en possession d'un article défectueux. Les entreprises touristiques produisent leurs services "live"+ devant le client. Cette situation contribue intrinsèquement à influencer le niveau des standards de qualité.

L'évaluation du service est subjective. Les clients jugent de la qualité et de l'utilité des services reçus dans un établissement touristique selon leurs sentiments et leurs habitudes. Il devient alors difficile de mesurer la véracité de la satisfaction de la clientèle puisqu'ill est, à la fois, difficile de déterminer les critères d'évaluation eux-mémes.

L'implantation du service nécessite des capitaux importants. Comparativement à plusieurs entreprises du secteur des services, l'implantation d'un hôtel exige un apport de capitaux beaucoup plus important. Etant donnd les contraintes exerces par les caractéristiques dont nous venons de faire mention, il est important que chacun des projets présentés aux investisseurs fasse l'objet d'une étude très approfondie.
Ces derniers ne retiendront alors que les projets offrant le plus de chances de réussite.

Compte tenu de ces facteurs et du rôle prépondérant du facteur humain dans cette industrie, nous verrons de quelle facon ces 6 facteurs et le rôle prépondérant du facteur humain dans cette industrie influenceront le processus de financement des projets.

\section{Financement conventionnel}

Il existe deux categories de capitaux dans une entreprise, les capitaux propres et les capitaux d'emprunt. Les premiers n'ont pas à être remboursés réguliêrement en conséquence, ils ne pesent pas de façon permanente sur la trésorerie de l'entreprise. Les seconds se distinguent des premiers par leurs echeanciers de remboursement: à demande provisoire, à court, moyen et long termes.

Les banques à charte canadiennes sont impliquées dans le financement des entreprises depuis longtemps. Toutefois, l'obtention d'un emprunt dans le secteur hôtelier ou touristique n'est pas aussi simple qu'il le semble. Chaque demande est étudié cas par cas et le dossier doit ètre complet afin d'obtenir l"approbation finale.

Cette réticence à financer des projets hôteliers provient du fait que l'hôtellerie et le tourisme d'une façon plus générale, sont considéres comme une "industrie tisquece" . Au grés des fluctuations de l'économie, cette perception est plus ou moins accentuée. Une etude ${ }^{\prime \prime}$ réalisée aupres de 32 directeurs de crédit américains en 1975 a permis de recueillir des commentaires tres significatifs, à ce sujet: " "...en autant que nous sommes concernés, les prêts que nous consentons aux hôtels sont considéres comme étant le type d'investissement le plus risqué que nous pouvons faire..." Heureusement, la perception des prêteurs aujourd'hui n'est pas aussi radicale. Soulignons l'engagement d'organismes gouvernementaux dans le développement et le financement de certains projets touristiques. 
De façon générale, l'analyse d'une demande de crédit dans une banque à charte sera jugée selon quatre critères principaux. Les trois premiers ont trait à la capacité de remboursement tandis que le quatrième se rapporte aux garanties exigibles en cas d'incapacité de remboursement.

Risque d'affaires. Le risque d'affaires tient essentiellement à la nature mème des activités de I'entreprise. Une entreprise représente un risque d'autant plus grand que sa structure de coût comporte plutôt des frais fixes, qu'elle oeuvre dans une industrie risqués, qu'elle est jeune et qu'elle en est a son premier prêt. Les banques ne sont pas des prêteurs de capitaux de risque. Elles sont prudentes face à certains types d'entreprise, notamment ceux du secteur touristique.

Une recherche réalisée par l'auteur auprès des succursales de banques à charte au Québec a permis de recueillir des informations utiles pour les éventuels emprunteurs. En voici quelques-unes:

Des les premiers contacts, il faut s'efforcer d'établir un climat de confiance avec le prêteur. L'expérience et une compréhension partagées de l'industrie touristique sont des composantes essentielles de la relation préteur/emprunteur. Un responsable de crédit sera moins intéressé de traiter avec un candidat dont la présentation du dossier exige beaucoup de temps.

L'expertise de gestion de l'emprunteur de mème que le nombre d'annes d'operation de l'entreprise sont des elements importants de l'évaluation du risque d'un prêt. L'analyse financière portera entre autres sur la structure d'endettement, la situation de l'encaisse et les autres engagements financiers de l'emprunteur. On lui demandera très souvent de joindre à son dossier des informations telles que le but de l'emprunt, l'étude de marché et le plan de remboursement. A noter que les conditions rattachées à l'obtention d'un prêt peuvent varier d'une succursale à l'autre.

Le personnel des succursales spécialisées dans les affaires commerciales possède habituellement plus d'expérience dans le crédit commercial. Le directeur de crédit ayant de l'ancienneté dispose également d'une plus grande marge d'autorisation de crédit. Si la demande doit être acheminée à un niveau hiérarchique supérieur, la crédibilitế du responsable de crédit à l'intérieur de l'institution joue un rôle primordial. Certains d'entre eux sont évalués à partir de critères tels que le nombre d'appels commerciaux réalisếs pendant l'année, le nombre de nouveaux comptes ouverts, le montant des prêts consentis et le résultat global de la gestion de leur portefeuille de prêt.

Risque financier. Le risque financier et les facteurs tels que la rentabilité et la struc-

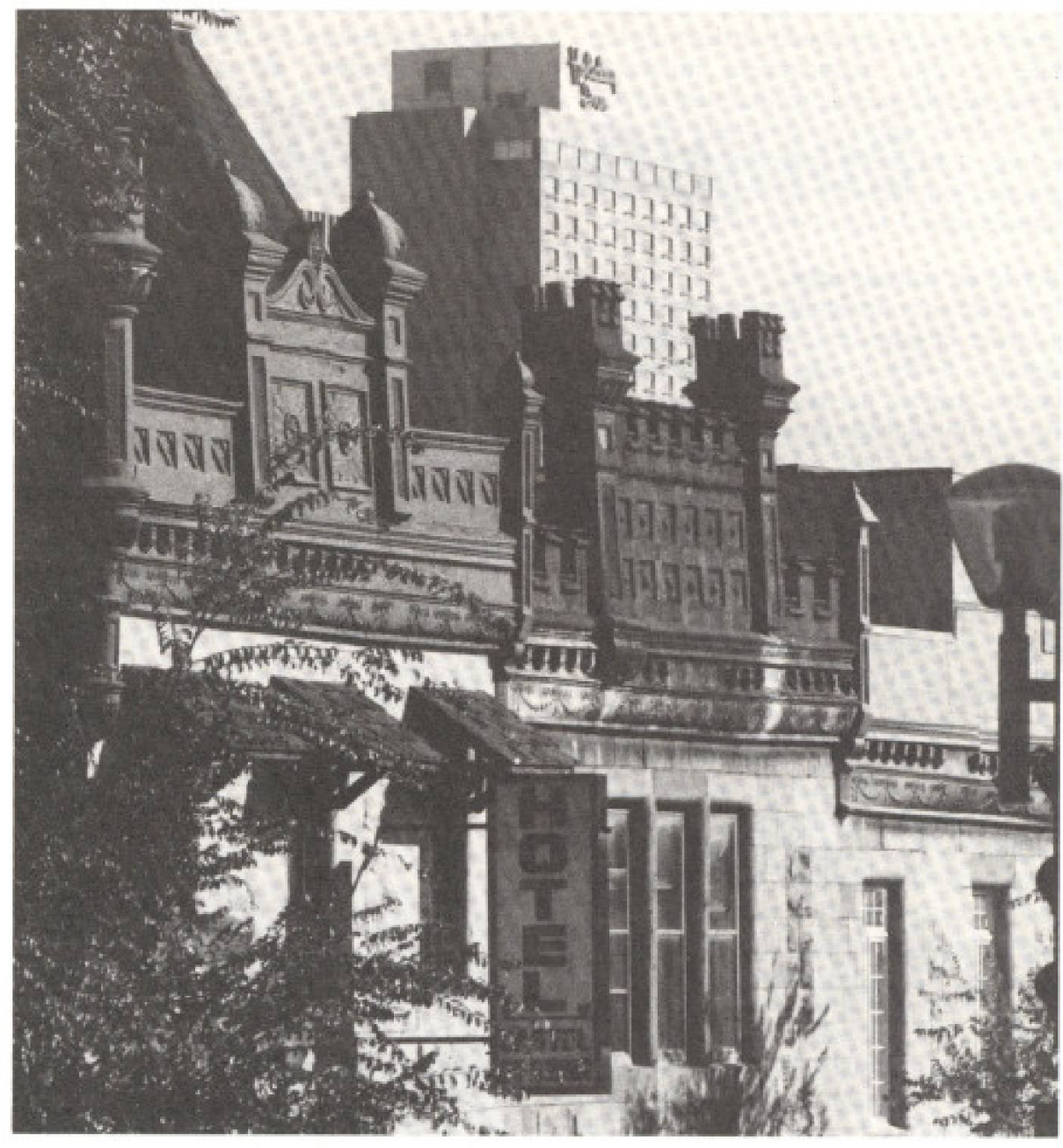

\section{L'expérience hôtelière}

ture d'endettement d'une entreprise sont les plus susceptibles d'affecter sa capacité de remboursement. À cette étape, le projet sera analysé froidement à partir d'une batterie de ratios financiers.

Risque managerial. Le risque managerial prend en compte l'expérience, la compétence, le savoir faire et les valeurs morales des gestionnaires de l'entreprise. Les gens moins expérimentés, les anciens faillis, les nouveaux propriétaires et même les nouveaux clients représentent plus de risques pour les banquiers.

Garanties. Le prêteur s'intéresse aux garanties offertes dans la mesure où il veut s'assurer qu'il pourra récupérer son prêt. Ce qui importe, c'est que le prêt ne viennent pas en défaut et que l'entreprise puisse le rembourser à même les revenus qu'elle génère dans les délais prévus. Dans le cas contraire, cela implique des frais d'administration acerus et une réduction des profits de la banque.

Les quatre criteres précedents sont interdépendants. Ils ont, l'un par rapport à l'autre, un effet multiplicateur ou compensatoire. Toutefois, si l'un d'entre eux ne satisfait pas aux exigences de la banque, le prêt n'est généralement pas accordé.
Plusieurs projets touristiques ou d'infrastructures hôtelières sont encore ou ont été financées par ce canal. Cependant, un nombre de plus en plus grand de promoteurs tente d'obtenir des capitaux à meilleur taux et pour ce faire, fait appel au public. Nous expliquerons maintenant les caractéristiques de ce type de financement.

\section{Financement public}

Au cours des dernières années, plusieurs facteurs ont contribué à l'émergence de nouveaux modes de financement. Les effets de la récession économique du début des années ' 80 , la diminution du taux d'inflation, les initiatives gouvernementales notamment en ce qui a trait a l'aide au développement touristique, au REA, aux mesures fiscales, à la capitalisation des entreprises et au décloisonnement des institutions financières sont autant de facteurs qui ont incité des promoteurs à développer de nouvelles formules de finanoement pour leurs projets. Ces formules se distinguent entre autres par le fait que le promoteur est appelé à traiter avec plusieurs petits investisseurs alors qu'auparavant, il n'avait bien souvent de relations qu'avec un investisseur principal. On a en quelque sorte assisté à une "désinstitutionnalisation" du financement. 
Sans entrer dans les technicalités du financement public, il sera question des trois formules les plus fréquemment utilisées à I'heure actuelle pour le finanoment de projets d'hôtels, soit la copropriété en commandite, le REA et lạ copropriété. Ces trois formules font en sorte que les projets sont finances avec l'argent des autres.

\section{Société en commandite}

La société en commandite est une méthode populaire et innovatrice pour obtenir des capitaux. Grâce à elle, les investisseurs peuvent acquérir une participation dans un projet offrant un bon potentiel économique tout en bénéficiant d'avantages fiscaux habituellement non disponibles dans d'autres véhicules financiers négociés publiquement. Après avoir livré le projet, le promoteur détient une participation et souvent il obtient le contrat de gestion de l'hôtel. La société en commandite vient compléter sa mise de fonds.

La mise en oeuvre d'une société en commandite exige toutefois la collaboration de nombreux professionnels, notamment des conseillers en administration spécialisés en hôtellerie et en tourisme, des avocats, des comptables, des courtiers, etc... Il va sans dire que cette opération est longue, frustrante à bien des égards, et très coûteuse. Toutefois, dès que la première transaction a été complétée et si l'opération a ćté bien menée par des spécialistes dans le domaine, les bénéfices tangibles ne tardent pas à venir.

\section{Régime d'épargne-actions}

De plus en plus de promoteurs de projets hoteliers ont fait ou considèrent faire usage du régime d'épargne-ăctions pour financer leurs projets. Cette formule de financement nécessite la collaboration de plusieurs professionnels et les coûts préalables à l'obtention des capitaux font en sorte qu'elle n'est pas accessible à tous.

Au cours des dernières semaines, la publicité a entouré de manière insistante diverses émissions de REA. On parle de surévaluation de certains titres, de réajustements prévisibles. On å même retiré certaines émissions du marché, faute d'acheteurs, En fait, plusieurs specialistes dans le domaine le confirment, la loi de l'offre et de la demande a été faussée. Plusieurs petits investisseurs, après avoir réalisé rapidement des gains substantiels se sont lancés à l'assaut de nouvelles émissions subjugués à l'idée de réaliser d'autres gains et de bénéficier d'avantages fiscaux. Toutefois, ils ont peut-être oublić qu'ils ont acheté des actions et non des obligations d'épargne. Il est donc à prévoir que le temps fera son oeuvre et que l'offre et la demande s'équilibreront.

Les promoteurs deprojets sérieux et bien structurés auront encore recours à cette formule, mais il semble que dans l'avenir, les investisseurs seront plus critiques et plus sélectifs avant d'investir. On remarque que l'injection de sommes d'argent dans des projets hôteliers est de plus en plus considérée par les investisseurs comme faisant partie d'une stratégie de diversification de leurs portefeuilles. Nous croyons que ce nouveau comportement ne pourra qu'être bénéfique à notre industrie.

\section{Copropriété}

La copropriété est un mode de financement plus specifique à I'industrie hôtelière ${ }^{2 !}$. Cette méthode permet à l'exploitant de bénéficier d'une capacité d'hébergement accrue sans avoir à en faire directement les frais. C'est d'ailleurs son principal avantage, surtout lorsqu'on songe aux nombreuses fluctuations salsonnières de cette industrie.

Chacune des unités d'hébergement en copropriété est financée par un investisseur privé dont le but principal n'est pas tant de réaliser un rendement financier sur son placement, mais plutôt d'être l'usufruitier de son placement. En d'autres mots, il désire obtenir le droit d'utiliser sa résidence secondaire (son unité) en échange d'un rendement éventuel.
$L^{+}$hôtelier ou une société de gestion peut également percevoir des honoraires de gestion relatifs à la location des unités et profiter au besoin d'unités d'hébergement additionnelles.

Le succes de cette formule exige toutefois la collaboration de plusieurs professionnels et un montage minutieux.

\section{Conclusion}

L'évolution du financement de projets touristiques au cours des dernières annees, a surtout été caractérisée par la combinaison de nombreux outils et véhicules financiers ct par l'ingéniosité démontrée dans la structuration financière des projets.

Comparativement aux modes de financement conventionnels, le processus d'obtention des capitaux lors des appels publics est beaucoup plus coûteux et implique la collaboration de plusieurs professionnels. D'autre part, le promoteur doit s'habituer à traiter avec plusicurs petits investisseurs contrairement à un seul comme cétait souvent le cas dans le passé.

Pour les promoteurs et les investisseurs, les projets les plus intéressants sont ceux qui genèrent par eux-mêmes des revenus. acquièrent une plus-value et finalement dont le montage financier est le plus effectif. Dans l'avenir, ni l'inflation, ni les avantages fiscaux ne suffiront à pallier aux mauvaises décisions d'investissement, mais les bons projets trouveront toujours à se financer. $f$

\section{Sources}

I1) W. Earl Sasser et Robert L. Banks, "Lender Artirs do Toward Hod Finucing ". The Cornell H.R.A. Quarterly, féviter 1976.

(2) Gilles Lariviere, associd de Laventhol \& Horwath. Allocution prononcéc au congriss de l"Association des hỏeliers du uébec. octobre 1986

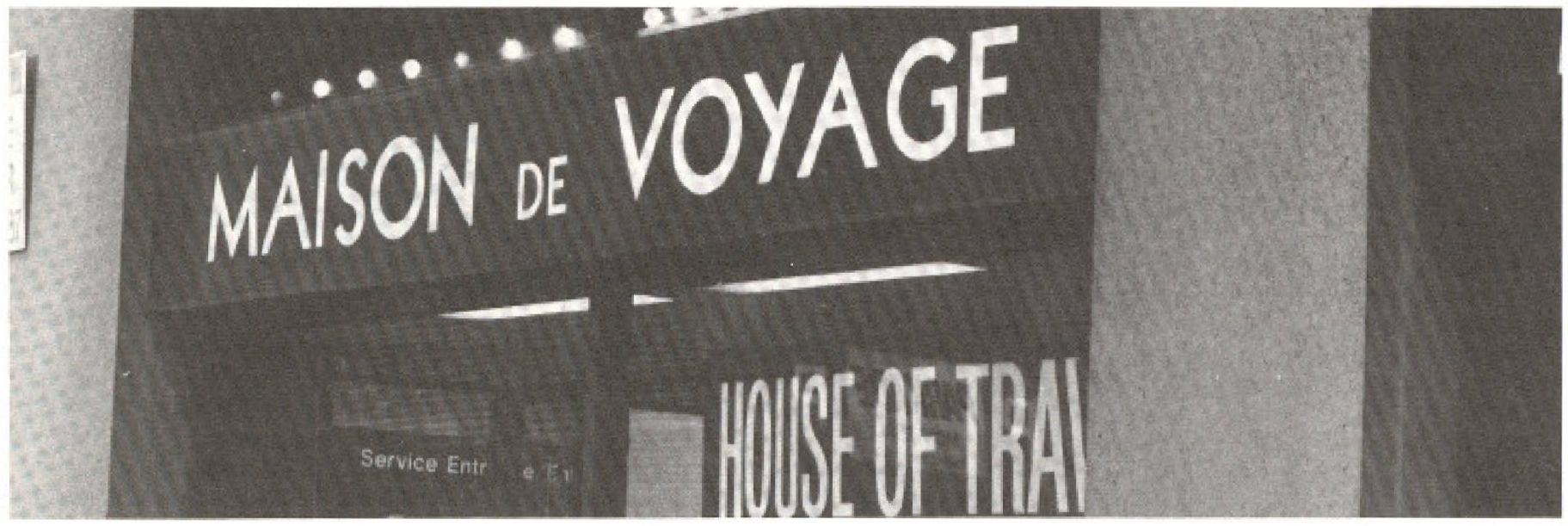

Des petites et très petites entreprises 